\title{
Lightweight Collaborative Filtering Method for Binary-Encoded Data
}

\author{
Sholom M. Weiss and Nitin Indurkhya \\ IBM T.J. Watson Research Center \\ P.O Box 218, Yorktown Heights, NY 10598, USA \\ sholom@us.ibm.com, nitin@data-miner.com
}

\begin{abstract}
A lightweight method for collaborative filtering is described that processes binary encoded data. Examples of transactions that can be described in this manner are items purchased by customers or web pages visited by individuals. As with all collaborative filtering, the objective is to match a person's records to customers with similar records. For example, based on prior purchases of a customer, one might recommend new items for purchase by examining stored records of other customers who made similar purchases. Because the data are binary (true-or-false) encoded, and not ranked preferences on a numerical scale, efficient and lightweight schemes are described for compactly storing data, computing similarities between new and stored records, and making recommendations tailored to an individual.
\end{abstract}

\section{Introduction}

Recommendation systems provide a type of customization that has become popular on the internet. Most search engines use them to group relevant documents. Some newspapers allow news customization. E-commerce sites recommend purchases based on preferences of their other customers. The main advantages of recommendation systems stem from ostensibly better targeted promotions. This promises higher sales, more advertising revenues, less search by customers to get what they want, and greater customer loyalty.

Collaborative filtering [1] is one class of recommendation systems that mimics word-of-mouth recommendations. An related task is to compare two people and assess how closely they resemble one another. The general concept of nearest-neighbor methods, matching a new instance to similar stored instances, is well-known [2]. Collaborative filtering methods use this fundamental concept, but differ in the how data are encoded, how similarity is computed, and how recommendations are computed.

We describe a lightweight method for collaborative filtering that processes binary-encoded data. Examples of transactions that can be described in this manner are items purchased by customers or web pages visited by individuals. As with all collaborative filtering, the objective is to match a person's records to customers with similar records. For example, based on prior purchases of a customer, one might recommend new items for purchase by examining stored 
records of other customers who made similar purchases. Because the data are binary (true-or-false) encoded, and not ranked preferences on a numerical scale, efficient and lightweight schemes are described for compactly storing data, computing similarities between new and stored records, and making recommendations tailored to an individual. Our preliminary results are promising and competitive with published benchmarks.

\section{Methods}

The collaborative filtering problem we looked at is a generic task that occurs in many applications. A specific example is recommending pages to a user surfing the web. We shall describe our algorithm within the context of this application where the attributes are the pages visited by users (and so we shall use "page" and "attribute" interchangeably). The value-attribute for a user simply records whether or not the user visited the corresponding page. Hence the data we are concerned with is purely binary.

In the simplest scoring scheme, recommendations might be made based on a linear weighted combination of other people's preferences. Most popular are memory-based methods. Complex model-based approaches such as Bayesian networks have also been explored [3]. We examined variations of a far simpler scheme. The basic idea is as follows:

1. find the $\mathrm{k}$ nearest neighbors to the new (test) case

2. collect all attributes of these neighbors that don't occur in the test case

3. rank these attributes by frequency of occurrence among the k neighbors.

In measuring distance between cases, we compute a score that measures similarity, the higher the score, the greater the similarity. For each training case, count the number of positive attributes in common with the test case. We add a small bonus for each page: the reciprocal of the number of training cases in which this page appears. This ensures that rare pages get a higher bonus than "popular" pages. As an example, suppose a new example has visited 5 webpages. We look at the stored examples to find similar examples. The most similar examples would match in all 5 webpages. Their scores would be 5 plus the value of the pre-computed bonuses for each of those pages. The score would ignore any negative distance that could be computed from pages visited in the stored examples but not visited by the new example.

Further improvements might be obtained by modifying step 2 slightly and splitting an example's vote. Instead of considering all attributes of all neighbors equally, we instead assign 1 vote for each neighbor, and split that vote among its attributes. The intuition behind this is that if a neighbor makes only one recommendation, it is more important than a recommendation made by a neighbor that makes 10 recommendations. This affects the frequency of occurrence and alters the ranking of the recommended attributes in the ranked list.

Another change we consider is to also measure the degree of similarity among neighbors' scores. Attributes of closer neighbors might be assigned a higher 
weight than those far away. We do this by using a weight of 1 for the closest neighbor(s), and a proportionally smaller weight for neighbors further away. The weight is the ratio of the score of a case to the highest score.

The overall algorithm is shown in Fig. 1. It follows the 3 steps listed earlier. Scoring a case involves computing a function $p v(j)$ for each attribute. This function measures the apriori predictive value of the attribute and is computed once at the start. Note the two modifications to the basic algorithm corresponding to vote splitting and relative similarity.

In order to compute the scores and tally the frequencies efficiently, the sample of cases are stored as follows:

- Case List. Here the cases are stored sequentially as a series of numbers corresponding to the positive attributes in the case. All cases are stored in a single vector, with another vector pointing to the start of each case. Table 1 shows an example of how the cases are implemented using two lists. The first case consists of positive attributes 2 and 5 . The second case consists of positive attributes $1,7,21$ and 43 . The third case begins with the attribute 2.

Table 1. Example of case list implementation

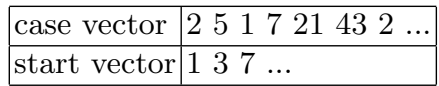

- Inverse List. Here we record a series of numbers corresponding to the cases in which a specific positive attribute occurs. All attribute mappings to examples can be stored sequentially in a single vector, with another vector pointing to the start of each attribute. Table 2 shows an example of how this is done. The first attribute occurs in cases 2 and 50, the second attribute occurs in cases 1,3 and 45 .

Table 2. Example of inverse list implementation

\begin{tabular}{|l|l|l|}
\hline inverse vector & $2501345 \ldots$ \\
\hline attribute start vector & $136 \ldots$ \\
\hline
\end{tabular}

The case list is used to compute the frequencies; the inverse list is used to compute the scores. Both the lists are computed once, at the start. Following that, the scores and frequencies can be computed very efficiently. 
Input: $C$ new case represented by $M$ attributes $C(1), \ldots C(m)$,

$\mathrm{D}$ \{Historical data of $\mathrm{n}$ cases, D1 ... Dn\}

Output: A \{Ranked list of attributes\}

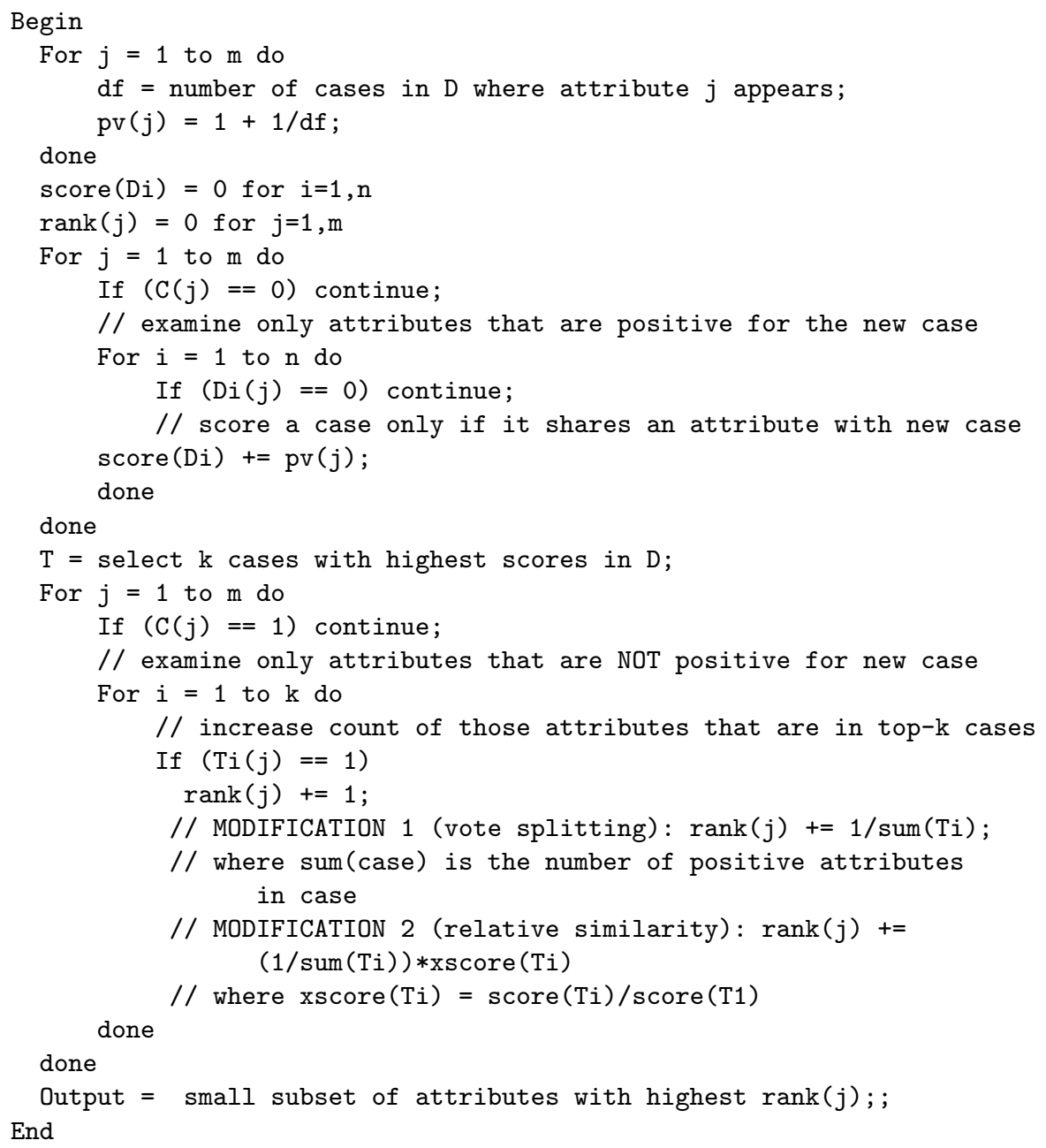

Fig. 1. The lightweight collaborative filtering algorithm 


\section{Results}

Our work is a followup to [3] which reports on empirical results using Bayesian networks and memory-based methods for three datasets. Our experiments were performed on the msweb dataset.

The problem we looked at is a generic task that occurs in many applications: recommending pages to a user surfing the web. The msweb data of users visiting pages at the Microsoft website can be viewed in this manner. The data is predivided into 32711 training cases and 5000 test cases, and 298 attributes. A case can be seen as a user and an attribute can be seen as a webpage visited by the user. 3] report a variety of experiments in which some attributes in the test cases are predicted by models based on the training data. One of the scenarios is as follows: in each test case, a visited page is randomly selected and "hidden". Based on the other attributes, the models learned from the training data attempt to recommend pages to visit. The models are evaluated by assessing how well they do in predicting the "hidden" page. Since our experiments require that test cases have 2 or more visited pages, the test cases used are a subset of the full set -3453 cases.

Since the models typically make a ranked list of recommendations, [3] use an R-metric to measure the quality of the recommendation. The R-metric is specified in Equations 1 and 2. Here, $R_{a}$ is the expected utility measuring how likely it is that the user will visit an item on the ranked list, with an exponential decay in likelihood for successive items, and is based on the user $a$ 's votes $v_{a, j}$, on item $j . R_{a}^{\max }$ is the maximum achievable utility and normalizing with it helps us consider results independent of sample sizes and the number of recommendations made. The higher the rank of the "hidden" page in the list of recommendations, the higher the value of the R-metric.

$$
\begin{gathered}
R_{a}=\sum_{j} \frac{\max \left(v_{a, j}-d, 0\right)}{2^{(j-1) /(\alpha-1)}} \\
R=100 \frac{\sum_{a} R_{a}}{\sum_{a} R_{a}^{\text {max }}}
\end{gathered}
$$

Table 3 summarizes the results obtained for several Model-based and Memory-based methods. The static ranked list simply recommends based on popularity and without considering the known votes. As such it serves as a baseline. Bayesian Clustering and Bayesian Networks are relatively complex Bayesian models [4]. The Correlation method [5] is further enhanced by the use of inverse user frequency, default voting and case amplification. Vector similarity [6] are enhanced by the use of inverse user frequency transformations as well. The three versions of the lightweight algorithm differ in the rank computation as described in Fig. 1 Except for the two Bayesian methods, all the other methods are memory-based methods.

The results in Table 3 are for the best variation of each method. Clearly the parameters of each method need to be optimized carefully. The performance of the lightweight method depends on $\mathrm{k}$, the number of neighbors used. Fig. 3 
Table 3. Summary of results on MSWEB data

\begin{tabular}{|c|c|}
\hline Collaborative Filtering Method & R-metric \\
\hline Static Ranked List & 49.77 \\
\hline Bayesian Clustering & 59.42 \\
\hline Vector Similarity & 61.70 \\
\hline Correlation & 63.59 \\
\hline Bayesian Network & 66.69 \\
\hline Basic Lightweight & 61.76 \\
\hline Lightweight with vote splitting & 64.35 \\
\hline Lightweight with vote splitting and relative similarity & 64.60 \\
\hline
\end{tabular}

Table 4. Top x recommendations

\begin{tabular}{|c|c|c|c|}
\hline $\mathrm{x}$ & 1 & 5 & 10 \\
\hline accuracy & .3165 & .7387 & .8412 \\
\hline
\end{tabular}

shows the result of varying $\mathrm{k}$ for each of the three variations. For a vote-splitting strategy, higher values of $\mathrm{k}$ are helpful. For the basic algorithm,, beyond a lesser number of $\mathrm{k}$, the performance gains are marginal.

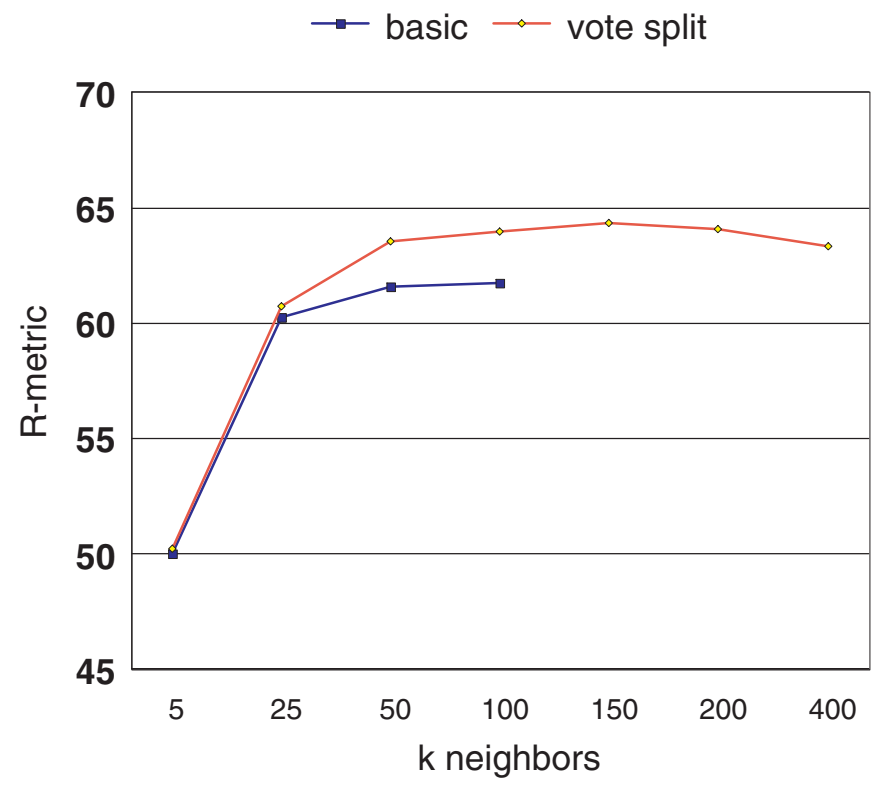

Fig. 2. Effect of $\mathrm{k}$ on performance of the lightweight method 


\section{Discussion}

The experiments suggest that our lightweight collaborative filtering method can be competitive with published results for far more complicated models. A lightweight algorithm, analogous to our basic collaborative filtering algorithm, has been extensively tested for information retrieval and document matching [7. An earlier study showed that in an IR environment, where more than one recommendation is made, simpler algorithms can be surprisingly effective [8].

We relied on published results for the alternative, highly specialized collaborative filtering methods. Our method is restricted to applications that are represented in binary form. Most real-world collaborative filtering methods expect ranked data, where a user is asked to rate products, for example on a scale of 1 to 5 . Far fewer benchmark datasets for binary data are publicly available. In the future, we can expect interest in binary representations to increase because greater automation is achieved without users manually assigned ratings.

The paucity of publicly available datasets with binary encodings limited our evaluation to one well-known dataset. Clearly, this is a weakness of the evaluation of our method. In the original study 3], several scenarios were examined including fixing the number of given items to 2,5 or 10 , each simulating different levels of information. Still, these variations are all taken from the same data, and the simulations create an unnatural set of data, all with the same number of positive features. We chose to use the one testing variation, all-but-one, that encompasses the variable scenarios of the unmodified snapshot of users.

Because memory-based methods, like our lightweight method, have complexity $\mathrm{O}(\mathrm{n})$, where $\mathrm{n}$ is the number of examples, many researchers have shifted attention from instance-based techniques to model-based techniques [9] 10 . We are not presenting our method as the best or as the most run-time efficient under all circumstances. Belief and dependency networks have many desirable properties, along with issues of complex representations and training. Our lightweight method is trivial to implement, mostly processes vectors sequentially, and operates in an environment where almost every attribute is measured as zero. These conditions lend themselves to relatively efficient implementations until the number of instances grows very large. Even then, experimentation may show that predictive performance reaches a plateau at a relatively small sample size of $\mathrm{N}$ cases. For example, on this dataset, much more data were originally available, but experimental results did not improve much with more data 9]. The simplicity of the lightweight method along with its binary vector representation, and its application without formal training to derive an intermediate model, may make it a suitable for some recommendation systems, including those with very large numbers of attributes.

Collaborative filtering matches an individual's history to users with similar records. An alternative approach to recommendations is item-based [10]. There, the top matches of each item are independently precomputed and stored (as in book retailers like Amazon), and only this information is used for tailoring a recommendation. Such an approach is efficient, has shown some good results, but has less potential for personalized recommendations. 
Many collaborative filtering algorithms, including our lightweight algorithm, ignore the sequence order of user-actions. Alternative algorithms, such as Markov models, can capture such information, which potentially can yield improved performance [11]. However, such models can become extremely complex, especially in higher dimensions, where either a long sequence of actions is traced or the space of recommendations is very large.

The R-metric is effective in measuring performance over a ranked list of recommendations. It rewards those recommendations that are correct and highly ranked, and it penalizes those recommendations that appear at the bottom of the list. We used the R-metric to facilitate comparisons to published results. Other metrics can also be examined. We measured the accuracy of finding the "hidden" page among the top x recommendations. This is shown in Table 4 An advantage of this metric is that by examining the trend for different values of $\mathrm{x}$, one can get a good idea of the quality of the solution.

Like search engines, the lightweight collaborative filtering algorithm uses an inverted list for efficient processing of sparse data. It requires very little data preparation and has a compact codebase. As such, it may prove highly desirable for applications that naturally fit a binary-encoded data representation.

\section{References}

1. D. Goldberg, D. Nichols, B. Oki, and D. Terry. Using collaborative filtering to weave an information tapestry. CACM, 35(12):61-70, 1992.

2. T. Cover and P. Hart. Nearest neighbour pattern classification. IEEE Transactions on Information Theory, 13:21-27, 1967.

3. J. Breese, D. Heckerman, and C. Kadie. Empirical analysis of predictive algorithms for collaborative filtering. In Proceedings of the Fourteenth Conference on Uncertainty in AI, 1998.

4. D. Chickering, D. Heckerman, and C. Meek. A bayesian approach to learning bayesian networks with local structure. In Proceedings of the Thirteenth Conference on Uncertainty in AI, 1997.

5. P. Resnick, N. Iacovou, M. Suchak, P Bergstrom, and J. Riedl. Grouplens: An open architecture for collaborative filtering of netnews. In Proceedings of the ACM Conference on Computer Supported Cooperative Work, pages 175-186, 1994.

6. G. Salton and M. McGill. Introduction to Modern Information Retrieval. McGrawHill, New York, 1983.

7. S. Weiss, H. White, and C. Apté. Lightweight document clustering. In Proceedings of PKDD-2000, pages 665-672. Springer, 2000.

8. S. Weiss, B. White, C. Apte, and F. Damerau. Lightweight document matching for help-desk applications. IEEE Intelligent Systems, pages 57-61, 2000.

9. D. Heckerman, D. Chickering, C. Meek, R. Rounthwaite, and C. Kadie. Dependency networks for inference, collaborative filtering and data visualization. Journal of Machine Learning Research, 1:49-75, 2000.

10. G. Karypis. Evaluation of item-based top-n recommendation algorithms. Technical Report 046, Department of Computer Science, University of Minnesota, 2000.

11. M. Deshpande and G. Karypis. Selective markov models for predicting web-page accesses. In Proceedings of SIAM Data Mining Conference, 2001. 